\title{
Retraso mental de causa genética. Estudio observacional en una zona de salud
}

Publicado en Internet:

23-noviembre-2015

Montserrat Tirado Melero: montsetiradomelero@gmail.com

\section{Tirado Melero ${ }^{a}$, M. E. Milagro Jiménez ${ }^{b}$, Y. Romero Salasc, J. Galbe Sánchez-Venturac, C. Balagué Clemós ${ }^{d}$, G. Zarazaga Gemes ${ }^{c}$, M. Cebrián Gimeno ${ }^{c}$}

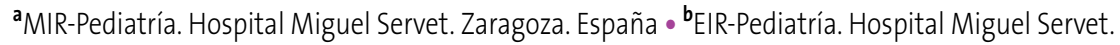
Zaragoza. España • 'Pediatra. CS Torrero La Paz. Sector II. Zaragoza. España • ' Enfermera de Pediatría.

CS Torrero La Paz. Sector II. Zaragoza. España.

Introducción: se realiza una revisión del número de trastornos del neurodesarrollo en la población pediátrica de un centro de Atención Primaria de la ciudad de Zaragoza (España), con el objetivo de conocer la prevalencia y la importancia creciente del diagnóstico genético.

Material y métodos: se realiza una búsqueda de casos en el programa de historia clínica informatizada OMI6 con los siguientes términos: "retraso mental", "retraso del desarrollo", "trastorno del desarrollo", "autismo".

Resultados: se han obtenido 76 casos de trastornos del neurodesarrollo, que son el 2,19\% de la población. Los diagnósticos principales fueron: 23 trastornos del lenguaje, 17 trastornos del aprendizaje, 11 de retraso mental, 10 casos de trastorno del espectro autista (TEA), 8 de retraso del desarrollo, 5 casos de dislexia, un caso de síndrome de Asperger y un caso de dispraxia motora. Se solicitó estudio genético en 21 casos, el $27,63 \%$ de los niños, siendo mucho más frecuente su realización en casos de retraso mental o TEA. Las pruebas genéticas proporcionaron el diagnóstico en 12 pacientes de los estudiados, un $57,14 \%$ de los casos. En general, se encuentra una causa genética en el $75 \%$ de los casos con diagnóstico establecido.

Retraso mental

- Trastorno del desarrollo

- Trastorno del espectro autista - Estudio genético

Conclusiones: este estudio muestra que los trastornos del neurodesarrollo aparecen en nuestra población con una frecuencia similar a la descrita en la bibliografía médica. Los estudios genéticos permiten cada vez más un diagnóstico etiológico preciso.

\section{Intelectual disability of genetic cause. Observational study in an urban primary health care center}

Introduction: we reviewed the number of neurodevelopmental disorders in the pediatric population of a primary care center in Zaragoza, in order to determine the prevalence and the growing importance of genetic diagnosis.

Material and methods: the research was carried out in the computerized medical record program OMI6 with the following terms: "mental retardation", "development delay", "development disorders" and "autism spectrum disorders".

Results: 76 cases of neurodevelopment disorders were found, which mean $2.19 \%$ of the population study. The main diagnoses were: 23 language disorders, 17 learning disorders, 11 mental retardation, 10 cases of autism, 8 development delay, 5 cases of dyslexia, 1 case of Asperger's syndrome and 1 case of dyspraxia. A genetic study was requested in 21 cases ( $27.63 \%$ of the children), being more frequent the implementation in cases of mental retardation or autism. The genetic test provided diagnosis in 12

Key words: patients of the study (57.14\% of the cases). A genetic cause was found in the $75 \%$ of the cases with an established diagnosis.

- Mental retardation

- Developmental delay

- Autism spectrum

Conclusion: this study shows that neurodevelopmental disorders appear in our population with a simdisorders

- Genetic tests ilar frequency as described in the literature. Genetic studies increasingly produce precise etiological diagnosis.

Cómo citar este artículo: Tirado Melero M, Milagro Jiménez ME, Romero Salas Y, Galbe Sánchez-Ventura J, Balagué Clemós C, Zarazaga Gemes G, et al. Retraso mental de causa genética. Estudio observacional en una zona de salud. Rev Pediatr Aten Primaria. 2015;17:309-15. 


\section{INTRODUCCIÓN}

Los trastornos del neurodesarrollo son alteraciones de la estructura neuroanatómica o de la función psicofisiológica que se pueden asociar con problemas cognitivos, académicos, conductuales, emocionales, sociales y del funcionamiento adaptativo. Este término engloba un gran número de patologías que se caracterizan por el deterioro de una o varias áreas del funcionamiento neurológico, provocando una deficiente adaptación al medio, de mayor o menor gravedad en función de la patología. Se pueden diferenciar dos grandes bloques: el de la discapacidad intelectual o retraso mental y el de los trastornos generalizados del desarrollo, incluyendo en este último los trastornos del espectro autista (TEA), trastornos específicos del lenguaje, la comunicación o el aprendizaje, entre otros.

El retraso mental se define como una capacidad intelectual significativamente disminuida, que se manifiesta en el periodo del desarrollo madurativo, y que provoca deficiencias del comportamiento adaptativo y limita la funcionalidad del individuo en la sociedad. Clásicamente se ha establecido el retraso mental como un cociente intelectual $(\mathrm{Cl})$ menor de 70, siendo el $\mathrm{Cl}$ medio de la población general de $100 \pm 15$ DS. Con estos valores obtenemos una prevalencia de retraso mental del $2-3 \%^{1}$, siendo las formas leves mucho más frecuentes. La tendencia actual es la de clasificar la discapacidad intelectual según el comportamiento del individuo en los dominios conceptual, social y práctico ${ }^{2}$; es decir, valorar las habilidades del niño en diferentes campos como el lenguaje, el aprendizaje, el razonamiento, la comunicación y participación social y el desempeño de actividades cotidianas.

El término retraso del desarrollo psicomotor o retraso global del desarrollo se reserva para niños menores de cinco años que no alcanzan los hitos del desarrollo a su edad correspondiente. En función de la gravedad predice discapacidad cognitiva o intelectual posterior, por lo que estos niños deben reevaluarse periódicamente. A partir de los cinco años pueden aplicarse diversos test para valorar la inteligencia y por ello a partir de esta edad ya se puede emplear el término discapacidad intelectual.

Los trastornos generalizados del desarrollo debutan en la primera infancia y pueden definirse como dificultades en la adaptación al medio por deficiencias en la comunicación, la interacción social, el lenguaje o el comportamiento que no pueden explicarse por una discapacidad intelectual u otras alteraciones neurológicas, motoras, sensoriales o de cualquier tipo. La incidencia actual de estos trastornos oscila entre un 20-30 por cada $10000^{3}$. El grupo más importante y más numeroso es el de Ios TEA, que cursan con deficiencias persistentes en la comunicación e interacción social, comportamientos y actividades restrictivas y repetitivas, que provocan un deterioro clínicamente significativo de lo social, escolar y demás áreas del funcionamiento habitual. También son frecuentes los trastornos del lenguaje, consistentes en dificultades persistentes en la adquisición y uso del lenguaje en cualquiera de sus modalidades, por fallo en la comprensión o la producción del mismo, lo que produce limitaciones funcionales importantes en la vida diaria.

Los pacientes con trastornos del neurodesarrollo deben ser estudiados en busca de un diagnóstico etiológico. Aunque no siempre se consigan resultados, la identificación de una causa mejora el manejo de estos niños en todos los niveles de asistencia, al poder obtener más datos sobre mecanismo de producción, pronóstico, tratamiento, otras alteraciones previsibles, posibles recurrencias, etc. Si bien la mayoría de estudios complementarios para llegar a un diagnóstico probablemente se van a llevar a cabo en una consulta especializada, generalmente la de Neuropediatría o la de Genética, el pediatra de Atención Primaria (AP) debe conocer las pruebas disponibles para saber qué recorrido va a seguir el niño y orientar a la familia sobre lo que va a suceder. También para los padres obtener el diagnóstico etiológico ayudará a resolver las preguntas que una patología de estas características sin duda plantea: ¿por qué ha sucedido esto?, ¿se podría haber evitado o detectado antes?, ¿existe tratamiento?, ¿cómo va a ser el desarrollo?, ¿puede 
volver a suceder en futuros hijos? No todas se pueden contestar con facilidad, pues tener una causa no asegura la evolución. A pesar de ello, el objetivo es llegar a un diagnóstico específico para comprender lo mejor posible el problema al que nos enfrentamos ${ }^{1}$. No existe un acuerdo sobre las pruebas necesarias para el diagnóstico, dada la amplia variedad de las mismas. En los últimos años, los adelantos en el campo de la genética molecular han permitido realizar muchos diagnósticos etiológicos que antes no eran posibles y es de esperar que a este respecto los avances sean aún mayores.

Con el objetivo de conocer la prevalencia de estos trastornos, haciendo hincapié en las causas y estudios genéticos de creciente importancia, se ha realizado un estudio descriptivo en un centro de AP de la ciudad de Zaragoza (España), con los pacientes correspondientes a los tres cupos de Pediatría del centro. Mediante una búsqueda informatizada se han extraído los casos de trastornos del neurodesarrollo, sus causas y las pruebas diagnósticas efectuadas, para determinar la frecuencia de diagnóstico etiológico y genético y conocer la rentabilidad de las técnicas de diagnóstico genético.

\section{MATERIAL Y MÉTODOS}

Se ha realizado una revisión del número de casos de trastornos del neurodesarrollo en un centro de AP urbano de la ciudad de Zaragoza (España), en la población asignada a los tres pediatras del mismo, que corresponde a 3472 niños a fecha 1 de agosto de 2014. La búsqueda se ha realizado en el programa de historia clínica informatizada OMI6 de la comunidad autónoma de Aragón, con los siguientes términos: "retraso mental", "retraso del desarrollo", "trastorno del desarrollo", "autismo". La búsqueda no excluye a los pacientes derivados a las consultas de Neuropediatría o Genética ni a Atención Temprana.

En cada uno de los casos encontrados se ha investigado, mediante el programa OMI6 o mediante el acceso a la historia clínica electrónica, además del sexo y la edad, si se había realizado o no estudio genético, los resultados del mismo y la presencia de otros estudios de interés para el diagnóstico etiológico del trastorno del desarrollo.

El acceso a las historias clínicas de los pacientes se hizo respetando las prescripciones legales y éticas al respecto.

\section{RESULTADOS}

Se han obtenido 76 casos de trastornos del neurodesarrollo, que son el 2,19\% de la población. Este porcentaje es acorde a lo que establece la bibliografía para estos trastornos. De ellos, 22 son niñas y 54 niños (28,95\% y 71,05\% respectivamente); también concuerda con la mayor frecuencia de estas patologías en el sexo masculino. La edad media de la población es de 8,3 años, con un mínimo de dos años y un máximo de 17 años.

Los diagnósticos principales obtenidos fueron: 23 trastornos del lenguaje, 17 trastornos del aprendizaje, 11 de retraso mental, 10 casos de TEA, 8 de retraso del desarrollo, 5 casos de dislexia, un caso de síndrome de Asperger y un caso de dispraxia motora (Fig. 1).

En 14 de los casos, un 18,42\% del total, consta algún diagnóstico secundario, entre los que se encuentran: trastorno por déficit de atención e hiperactividad (TDAH), epilepsia o retraso del crecimiento (Tabla 1).

Se encuentra un diagnóstico etiológico en el $21,05 \%$ de los casos, en 16 de los niños, presentando las siguientes causas: retraso del desarrollo por parálisis cerebral, retraso mental por hematoma epidural postraumático, trastorno del aprendizaje por un posible síndrome de alcoholismo fetal, trastorno del lenguaje por problemática social, síndrome de Down, síndrome de Dravet, síndrome de Cornelia de Lange (dos hermanos), síndrome de $X$ frágil y cromosomopatías por microalteraciones de los cromosomas (deleción subtelomérica en cromosoma 18q, exceso de material genético en la zona satélite del cromosoma 22, microdeleción en cromosoma 4 y microdeleción en cromosoma $22 q$ o síndrome de Phelan-McDermid). 


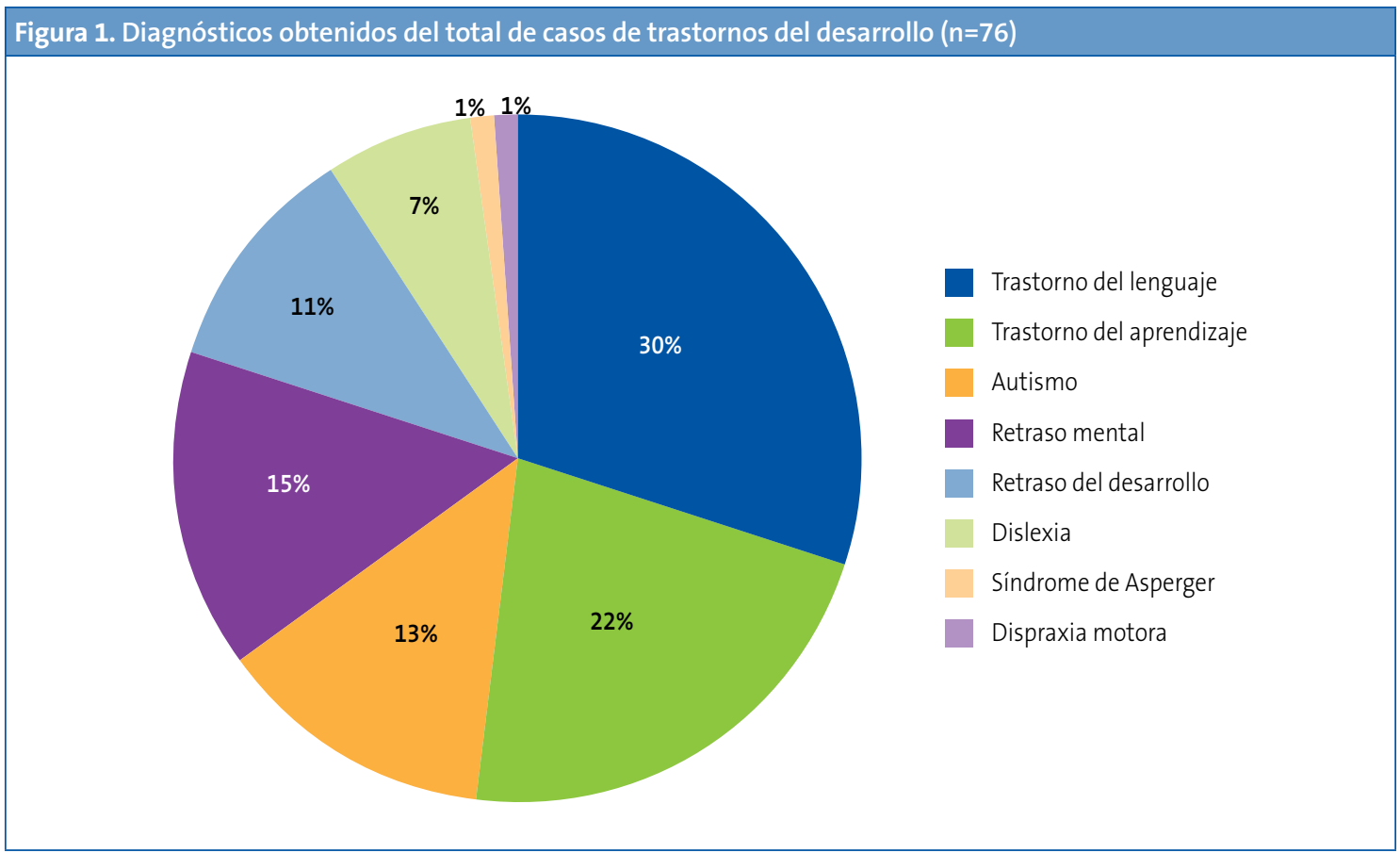

Se solicitó estudio genético en 21 casos, el 27,63\% de los niños, siendo mucho más frecuente su realización en casos de autismo o retraso mental que en los trastornos simples del lenguaje o del aprendizaje: en el 90,9\% de casos de retraso mental y en el $70 \%$ de casos de autismo, mientras que en el resto de diagnósticos solo se realizó en un 5,45\% de niños. Los estudios practicados fueron en todos los casos cariotipo de alta resolución, estudio genético de $\mathrm{X}$ frágil y array-CGH, además del estudio genético de síndrome de Dravet en el caso confirmado como tal. La única excepción a toda la batería de pruebas son los cuatro casos de síndrome de
Down, ya que ante la sospecha clínica clara, la única prueba necesaria fue el cariotipo de alta resolución. Las pruebas genéticas proporcionaron el diagnóstico en 12 pacientes de los estudiados, esto es un $57,14 \%$ de los casos. En nuestra población, se encuentra una causa genética en el 75\% de los casos con diagnóstico establecido.

\section{DISCUSIÓN}

Se trata de un estudio descriptivo elaborado en un centro de AP de la ciudad de Zaragoza, a partir de

\begin{tabular}{|l|c|c|c|c|c|c|}
\hline \multirow{2}{*}{ Tabla 1. Diagnósticos secundarios obtenidos en cada uno de los casos de trastorno del neurodesarrollo } \\
\cline { 2 - 8 } & \multicolumn{7}{|c|}{ TDAH } & TEA & Trastorno del lenguaje & Retraso del crecimiento & No consta & Casos totales \\
\hline Trastorno del lenguaje & 2 & 0 & 0 & 0 & 21 & 23 \\
\hline Trastorno del aprendizaje & 1 & 0 & 5 & 0 & 11 & 17 \\
\hline Retraso mental & 0 & 2 & 0 & 0 & 9 & 11 \\
\hline TEA & 0 & 0 & 1 & 0 & 5 & 10 \\
\hline Retraso del desarrollo & 2 & 0 & 0 & 1 & 5 & 5 \\
\hline Dislexia & 0 & 0 & 0 & 0 & 1 & 1 \\
\hline Síndrome de Asperger & 0 & 0 & 0 & 0 & 1 & 1 \\
\hline Dispraxia motora & 0 & 0 & 0 & 0 & & 1 \\
\hline
\end{tabular}

TDAH: trastorno por déficit de atención e hiperactividad; TEA: trastornos del espectro autista. 
los datos obtenidos del programa OMI6 y de la historia clínica electrónica, para conocer la prevalencia de trastornos del desarrollo y la proporción de diagnósticos etiológicos. Presenta algunas limitaciones, la más importante la posible pérdida de casos por estar registrados en OMI6 con alguna codificación diferente a aquellas con las que se ha realizado la búsqueda. También la pérdida de información, por la dificultad en este programa para codificar algunos diagnósticos específicos.

Se han comparado estos resultados con los de la Unidad de Neuropediatría del Hospital Universitario Miguel Servet de Zaragoza ${ }^{4}$. Debemos señalar que los casos de trastornos del neurodesarrollo de nuestro centro son derivados y estudiados en esta unidad. En ella se consigue un diagnóstico etiológico en el $31 \%$ de los casos, frente al 17,8\% del presente estudio. Se puede explicar por su mayor volumen de casos y por la falta de información en el programa OMI6. De sus casos con diagnóstico establecido, el $46 \%$ estaban genéticamente determinados. En este estudio se aprecia una causa genética en el $75 \%$ de los diagnósticos etiológicos. Estos porcentajes resaltan la importancia creciente de la genética en el diagnóstico de patología neurológica que hasta hace pocos años no tenía explicación. Aunque en algunos pacientes el fenotipo puede sugerir la presencia de cromosomopatía subyacente al trastorno del desarrollo, en otros el trastorno neurológico será el único presente; aun en estos casos la genética será fundamental, pues las alteraciones microscópicas de los cromosomas pueden producir una clínica exclusivamente neurológica. Es función del pediatra de AP conocer este hecho y conocer las técnicas que se llevan a cabo en las consultas de Genética, con el objetivo de informar a las familias de las posibles pruebas que se realizarán en los niños. Las técnicas que se realizan de manera más habitual son las siguientes:

- Cariotipo. Está universalmente establecido que el cariotipo se debe realizar de forma sistemática a todos los niños con retraso mental de causa no aclarada ${ }^{1}$. Se prefiere un cariotipo de alta resolución (que es el que identifica al menos 550 bandas), ya que además de las anomalías numéricas de los cromosomas, puede encontrar deleciones o adiciones de solo 5 Mb del ADN ${ }^{5}$.

- Hibridación in situ con fluoresceína (FISH). Consiste en asociar a un fragmento cromosómico o genético conocido una sonda fluorescente, para detectar la presencia o ausencia de ese fragmento en el ADN del paciente que estamos estudiando ${ }^{5}$. El inconveniente es que se debe sospechar la alteración a estudiar. Es la técnica más habitual para diagnosticar síndromes de microdeleciones como el síndrome de Di George, el de Prader-Willi o el de Williams.

- Estudio de anomalías subteloméricas. Estas zonas presentan una elevada tasa de recombinación que facilita la aparición de deleciones o duplicaciones; además son zonas que contienen un gran número de genes y por lo tanto pequeñas alteraciones del material genético pueden tener consecuencias graves. Estas regiones se pueden valorar mediante la técnica FISH de los telómeros de cada cromosoma, por multiple ligation probre amplification (MLPA) para detectar número de copias de genes, o por análisis de polimorfismos del ADN (detección de SNP).

- Estudios moleculares: array-CGH (hibridación genómica comparada). Es la herramienta más potente en la identificación de anomalías cromosómicas submicroscópicas. Son dispositivos compuestos de una superficie sólida en la que se han dispuesto miles de sondas de ADN, cada una de las cuales identificará un determinado ADN, ARN o polimorfismo genético ${ }^{6}$. La ventaja es que pueden detectar miles de genes simultáneamente y por ello son una buena herramienta para el estudio de trastornos del neurodesarrollo de causa no explicada. Algunos autores sugieren que se realice este estudio como primer paso en lugar del cariotipo en estos pacientes, y reservar el cariotipo para sospecha de síndromes cromosómicos clásicos o ante historia familiar de reordenamientos cromosómicos (ya que esta técnica no muestra translocaciones o inversiones balanceadas). 
El trastorno del desarrollo más prevalente en este estudio es el trastorno del lenguaje. No es un dato sorprendente, teniendo en cuenta que hasta el $15 \%$ de los niños en edad preescolar y un 5-9\% de los escolares tienen un trastorno del habla o el lenguaje $^{7}$. La codificación del programa OMI6 no permite distinguir si se trata de un retraso simple del lenguaje, de tipo madurativo y con buena evolución, o de un trastorno específico del lenguaje, de mayor gravedad y repercusión importante sobre el aprendizaje, el comportamiento o las habilidades sociales. En cualquier caso, la actuación del pediatra de AP debe ser la detección temprana del problema de lenguaje y la derivación a atención temprana y al logopeda, también de forma precoz para que su intervención sea lo más efectiva posible. En casos graves o si hay otros síntomas acompañantes, es conveniente derivar también a la consulta de Neuropediatría. La mayoría de niños con trastorno del lenguaje mejora sus capacidades comunicativas, aunque pueden persistir alteraciones hasta la vida adulta, y también se han relacionado estos trastornos con problemas para la lectura más tardíamente.

Los trastornos del desarrollo se pueden considerar globalmente un problema que está presente en las consultas del pediatra de AP, en algunos casos grave. En muchas ocasiones será el pediatra del niño el que detecte los signos de alarma en las revisiones de salud habituales. En otros, es la familia la que comunica sus sospechas, de ahí la importancia de prestar atención también a las preguntas o comentarios de los padres. Estos trastornos requieren una intervención precoz y multidisciplinar, por parte de pediatras, fisioterapeutas, logopedas, psicólogos y trabajadores sociales, entre otros especialistas. A este respecto las Unidades de Atención Temprana son un instrumento fundamental que integra múltiples cuidados y que supone además un apoyo importante a las familias de los niños con estos trastornos. Es por eso que la derivación a estos centros debe ser también precoz, ya que parte de la evolución del niño dependerá de la estimulación y los cuidados adecuados a su problema. Entre todas las consultas y pruebas, el pediatra de AP puede ser el punto de referencia para la familia, aportando información, detectando patología asociada, derivando a los servicios necesarios, informando de recursos existentes y de asociaciones de pacientes, y sobre todo ayudando a la familia a comprender mejor a su hijo para lograr la mejor calidad de vida posible.

\section{CONFLICTO DE INTERESES}

Los autores declaran no presentar conflictos de intereses en relación con la preparación y publicación de este artículo.

\section{ABREVIATURAS}

ADN: ácido desoxirribonucleico - AP: Atención Primaria - ARN: ácido ribonucleico $\bullet \mathrm{Cl}$ : cociente intelectual • DS: desviación estándar • FISH: hibridación in situ con fluoresceína - MLPA: multiple ligation probre amplification $\bullet$ TDAH: trastorno por déficit de atención e hiperactividad - TEA: trastornos del espectro autista.

\section{BIBLIOGRAFÍA}

1. Galán-Gómez E, Méndez-Pérez MP, Delgado-Rubio A. Estudios genéticos en el retraso mental inespecífico. An Pediatr Contin. 2012;10:7-15.

2. Asociación Americana de Psiquiatría. Guía de Consulta de los criterios diagnósticos del DSM 5. Arlington, VA: Asociación Americana de Pediatría; 2013.

3. García-Ron G. Indicadores clínicos precoces de los trastornos generalizados del desarrollo. An Pediatr (Barc). 2012;77:171-5.

4. López-Pisón J. Nuestra experiencia en el diagnóstico etiológico del retraso global del desarrollo y discapacidad intelectual: 2006-2010. Neurología. 2013;29: 402-7.

5. Moeschler J. Genetic evaluation of intelectual disabilities. Semin Pediatr Neurol. 2008;15:2-9.

6. Alonso J. Utilidad de los microarrays en Pediatría. An Pediatr Contin. 2008;6:218-22.

7. Gassió-Subirachs R. Trastornos del lenguaje. An Pediatr Contin. 2006;4:140-4.

8. Moeschler J, Shevell M. Clinical genetical evaluation of the child with mental retardation or development delays. Pediatrics. 2006;117:2304-13. 
9. López-Pisón J, Monge-Galindo L. Evaluación y manejo del niño con retraso psicomotor. Trastornos generalizados del desarrollo. Rev Pediatr Aten Primaria Supl. 2011;20:131-44.

10. Gónzalez-Meneses A. Dismorfología clínica y genética I: enfoque diagnóstico del paciente dismórfico. An Pediatr Contin. 2008;6:140-6.

11. Martínez Fernández ML, Bermejo E, Martínez Frías ML. Ejemplos clínicos de alteraciones crípticas de
ADN y guías para sospechar que un niño pueda tener alguna alteración críptica o molecular. SEMERGEN. 2010;36:573-8.

12. González-Alvaredo S, Sanz Rojo R, García Santiago J, Gaztañaga Expósito R, Bengoa A, Pérez-Yarza EG. Criterios de diagnóstico genético en casos de retraso mental y del desarrollo de origen idiopático. An Pediatr (Barc). 2008;69:446-53. 